\title{
Rehabilitación olfatoria en pacientes laringectomizados: maniobra de inducción del flujo aéreo nasal
}

\author{
Olfactory rehabilitation in laryngectomized patients: nasal airflow \\ inducing maneuver
}

Romina Ovalle A. ${ }^{1}$, Sebastián Sánchez J. ${ }^{2}$, Alfredo Urrutia F. ${ }^{1}$, Jaime Osorio M. ${ }^{1,3}$

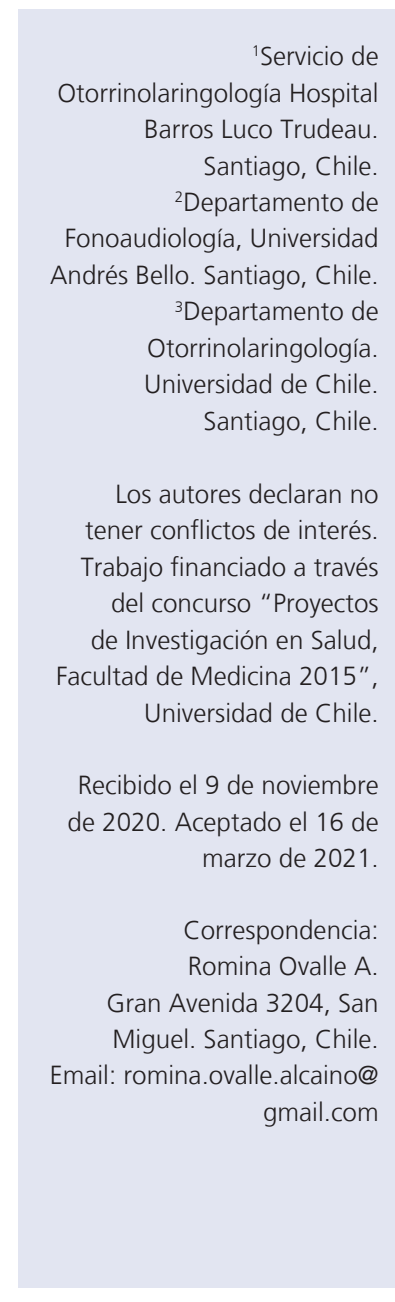

\section{Resumen}

Introducción: La laringectomía total (LT) tiene como secuela la perdida de la voz, pero otra consecuencia no estudiada es la pérdida del olfato. Objetivo: Demostrar que la "maniobra de inducción del flujo aéreo nasal" (MIFAN) rehabilita el olfato en pacientes con LT. Material y Método: Estudio cuasiexperimental antes-después en pacientes laringectomizados por cáncer de laringe del Servicio de Otorrinolaringología del Hospital Barros Luco Trudeau (HBLT) de Santiago de Chile. Evaluación a través de encuesta, examen físico, nasofibroscopía y test olfatométrico. Pacientes con alteración del olfato por transmisión serán enrolados y se enseñará la MIFAN. Resultados: Se estudiaron 12 pacientes: 10 hombres, 2 mujeres. Edad promedio 66,3 años, todos autovalentes. 66,6\% presentó anosmia y $33,3 \%$ hiposmia. Todos lograron realizar la maniobra. Posrehabilitación el 100\% presentó presencia de olfato valorada por olfatometría. Población intervenida similar a otras series en cuanto a sexo y edad. La erigmofonación facilita la rehabilitación con MIFAN. La rehabilitación del olfato se logró en todos y paralelamente mejoró el sentido del gusto. Conclusión: La MIFAN es una técnica sencilla, barata y asequible para lograr rehabilitar el sentido del olfato en pacientes laringectomizados. Palabras clave: Laringectomía total, anosmia, hiposmia, rehabilitación, olfato.

\begin{abstract}
Introduction: Total laryngectomy (TL) has as a consequence the loss of voice, but another not studied consequence is the loss of smell. Aim: To demonstrate that the "nasal airflow inducing maneuver" (NAIM) rehabilitates smell in patients with TL. Material and Method: A quasi-experimental before-after study in laryngectomized patients for laryngeal cancer from the Otorhinolaryngology Service (ENT) of the Barros Luco Trudeau Hospital (BLTH) at Santiago, Chile. Evaluation through survey, physical examination, nasofibroscopy and olfactory test. Patients with transmission impairment of smell were enrolled and NAIM was performed. Results: 12 patients were studied: 10 men, 2 women. Average age 66.3 years. All self-supporting. $66.6 \%$ presented anosmia and $33.3 \%$ hyposmia. They all managed to perform the maneuver. Post-rehabilitation, 100\% presented the presence of smell assessed by olfactometry. Intervened population similar to other series in terms of sex and age. Esophageal speech facilitates NAIM rehabilitation. Rehabilitation of smell was achieved in all of them and in parallel, the sense of taste improved. Conclusion: NAIM is a simple, cheap and affordable technique to rehabilitate the sense of smell in laryngectomized patients.
\end{abstract}

Keywords: Total laryngectomy, anosmia, hyposmia, rehabilitation, smell.

\section{Introducción}

La laringectomía total (LT) es una técnica quirúrgica utilizada mayoritariamente en pacientes con cáncer de laringe. Es una condición altamente incapacitante ya que altera todas las funciones de la laringe, siendo la perdida de la voz la consecuencia más evidente ${ }^{1-3}$. Sin embargo, otra consecuencia igual de importante es la pérdida del flujo aéreo nasal deter- 
minando la presencia de anosmia/hiposmia al no estimular el epitelio olfatorio del techo de las fosas nasales e indirectamente, por la relación estrecha entre el sentido del olfato y del gusto, los pacientes también presentan ageusia o disgeusia ${ }^{4}$.

La rehabilitación del paciente laringectomizado debe ser por tanto integral considerando todas las funciones que se pierden o alteran al extirpar la laringe $e^{5} . \mathrm{Al}$ analizar la literatura actual destaca el gran número de estudios enfocados a la rehabilitación de la voz, existiendo un limitado número de trabajos que aborden la rehabilitación del olfato en los pacientes laringectomizados. En Chile no existe ninguna experiencia en este sentido ${ }^{4,5}$.

Las alteraciones del olfato pueden ser de percepción cuando existe una alteración del epitelio o de las vías olfativas y de transmisión cuando la vía olfativa está intacta, pero existe alguna alteración que impide que las moléculas olorosas lleguen al epitelio olfatorio como ocurre en los pacientes con LT al interrumpirse el paso de aire por las vías aéreas superiores ${ }^{6}$. Para las alteraciones de transmisión del olfato algunos autores han desarrollado distintas técnicas para restablecer el flujo aéreo nasal como el by-pass laríngeo o la presión glosofaríngea, pero ninguna de ellas se encuentra incluida en los métodos rutinarios de rehabilitación del paciente laringectomizado ${ }^{4,5}$. En el año 2000, Hilgers y cols. describieron la maniobra de "nasal airflow inducing maneuver (NAIM)", traducido al español como: "maniobra de inducción de flujo aéreo nasal (MIFAN)" o conocida como "técnica del bostezo educado" . Existen algunos trabajos que han corroborado sus resultados, dentro de ellos el estudio de Morales-Puebla y cols. .

\section{Objetivo}

El objetivo principal de este trabajo es demostrar que la MIFAN logra rehabilitar el olfato en pacientes laringectomizados del servicio de Otorrinolaringología (ORL) del Hospital Barros-Luco Trudeau (HBLT). Los objetivos específicos son describir las características clínicas de los pacientes seleccionados y conocer la repercusión de la rehabilitación del olfato sobre el sentido del gusto.

\section{Material y Método}

Estudio cuasiexperimental antes-después en pacientes chilenos operados de LT por cáncer de laringe en controles regulares en el Servicio de ORL del HBLT hasta febrero del año 2016. Tamaño muestral por conveniencia. Aprobación por el comité de ética del Servicio de Salud Metropolitano Sur.

Antes de ingresar al estudio a todos los pacientes se les aplicó una encuesta sobre sus antecedentes clínicos y de su percepción del sentido del olfato y del gusto antes y después de la LT. Posteriormente fueron evaluados por otorrinolaringólogo y fonoaudiólogo para descartar alteraciones anatómicas en nariz y cavidad oral y/o alteraciones neurológicas (parálisis facial, hipotonías, deterioro cognitivo severo, entre otras) que pudieran interferir con la rehabilitación y en caso de existir descartarlos del estudio.

Luego se les aplicó el test olfatométrico cualitativo sniffin sticks screening test con tres aromas que fue validado para la población chilena en el año $2012^{7}$. Con esto dividimos a los pacientes en dos grupos, los con olfato y los sin olfato. Se volvió a repetir la misma prueba, pero ahora con un by-pass laríngeo (Figura 1) que permite comunicar el flujo aéreo naso-oral directamente al traqueostoma mediante un tubo corrugado, objetivando el paso de aire con un espejo de Glatzel. Al restablecer el flujo aéreo nasal permitía determinar que pacientes recuperaban el olfato. Si recuperaban el olfato ingresaban al estudio y el fonoaudiólogo les enseñaba inmediatamente la MIFAN explicando los ejercicios mandibulares y linguales.

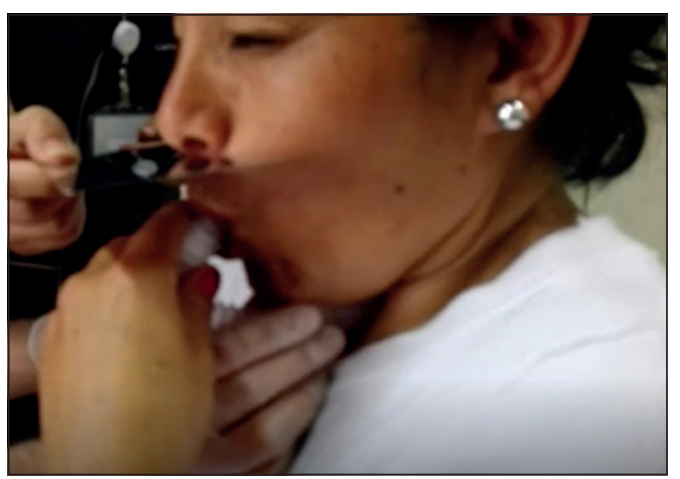

Figura 1. By-pass laríngeo. 
Si no lograban oler en ningún grado se asumía anosmia sensorial ya que pese a estar presente el estímulo no había respuesta por parte del neuroepitelio olfatorio. Estos pacientes se excluyeron del estudio.

La MIFAN consiste en movimientos de la mandíbula y la lengua que permiten generar una variación de presión, ya sea negativa o positiva, que se transmite desde la cavidad oral a las fosas nasales logrando así un flujo aéreo en las mismas. Con la LT se obtiene una vía aérea inferior totalmente independiente de la vía digestiva, por lo que al efectuar un movimiento de descenso de la mandíbula y la lengua con los labios sellados, se produce una presión negativa en la cavidad oral que se transmitirá a las fosas nasales, generando así la entrada de aire. Posteriormente y manteniendo los labios cerrados, se efectúa un movimiento de ascenso de la mandíbula y de la lengua hasta tocar el paladar lo que generará una presión positiva en la boca que expulsará el aire inhalado por las fosas nasales. La realización de estos movimientos repetidamente generará una corriente de aire suficiente para estimular el epitelio olfativo ${ }^{4,5}$.

Al mes de enseñada la técnica, el fonoaudiólogo reevaluó la correcta realización de la maniobra y corrigió errores en caso de existir. Por la rápida y correcta adquisición de la maniobra observada en este control se decide nueva evaluación a los 2 meses de enseñada la MIFAN aplicando nuevamente la encuesta de percepción del olfato y del gusto y el test olfatométrico para comparar resultados pre y posrehabilitación. Las variables fueron analizadas con el programa STATA 12 y test de Wilcoxon para variables categóricas no paramétricas.

\section{Resultados}

Se evalúo a 17 pacientes de los cuales se excluyeron a cinco: tres con alteraciones anatómicas (desviaciones septales severas), uno por no poder acudir a todos los controles (vivía fuera de la ciudad), y otro porque se consideró con anosmia sensorial. Los 12 pacientes restantes fueron enrolados y completaron la intervención. En cuanto al sexo, dos fueron mujeres y 10 hombres. Todos los pacientes eran autovalentes, con un promedio de edad de 66,3 años (52-74 años). Un 83\% recibió ra- dioterapia coadyuvante y un 16,7\% requirió de una reintervención quirúrgica con necesidad de colgajo. En cuanto a la rehabilitación de la voz un $41,6 \%$ contaba con técnica foniátrica.

La duración de la primera sesión de entrenamiento de la MIFAN fue variable para cada paciente teniendo como promedio 20 min (15-30 min), la que se dio por finalizada hasta comprobar que el paciente era capaz de realizar los movimientos bucales de forma adecuada con una nueva aplicación de aroma por fosas nasales. En todos se logró la técnica en la primera sesión y la mantuvieron durante el proceso.

Tras el primer test olfatométrico el 33,3\% (4/12) de los pacientes fueron capaces de percibir la presencia de un aroma, mientras que el 66,6\% (8/12) restante no tenían olfato. En la repetición de este test con el by-pass laríngeo, el $100 \%$ fue capaz de detectar la presencia de olor. Todos aquellos que identificaron la presencia de un aroma antes del by-pass laríngeo practicaban erigmofonación como técnica de fonación.

$\mathrm{Al}$ evaluar el olfato con el test olfatométrico antes y después de la rehabilitación (Figura 2), se observó que antes de la rehabilitación un $66,6 \%$ de los pacientes presentaban anosmia y un $33,3 \%$ hiposmia, mientras que después de la rehabilitación el $100 \%$ de los pacientes presentaron olfato, siendo esta diferencia estadísticamente significativa (test de Wilcoxon $\mathrm{p}=0,002)$.

Al evaluar los cambios en la percepción del olfato y del gusto antes y después de la rehabilitación valorados en la encuesta de percepción, observamos en relación al olfato, en una escala de $0 \%-100 \%$, que los pacientes percibían tener en promedio un $88 \%$ de olfato antes de la LT. Luego de la cirugía, este porcentaje bajó a un $26 \%$ y mejoró a un $60 \%$ luego de la rehabilitación. Lo mismo ocurre con el gusto, donde la percepción sensorial fue de un $97 \%$ antes de la cirugía, bajando a un $46 \%$ luego de esta, y posrehabilitación sube a un $58 \%$. Siendo ambas diferencias estadísticamente significativas (test de Wilcoxon olfato: $\mathrm{p}=0,0022$, gusto: $\mathrm{p}=0,015)$.

En la sección de "comentarios" de la encuesta, la valoración global de los pacientes respecto a la rehabilitación con esta maniobra fue buena. Los pacientes que tenían capacidad 


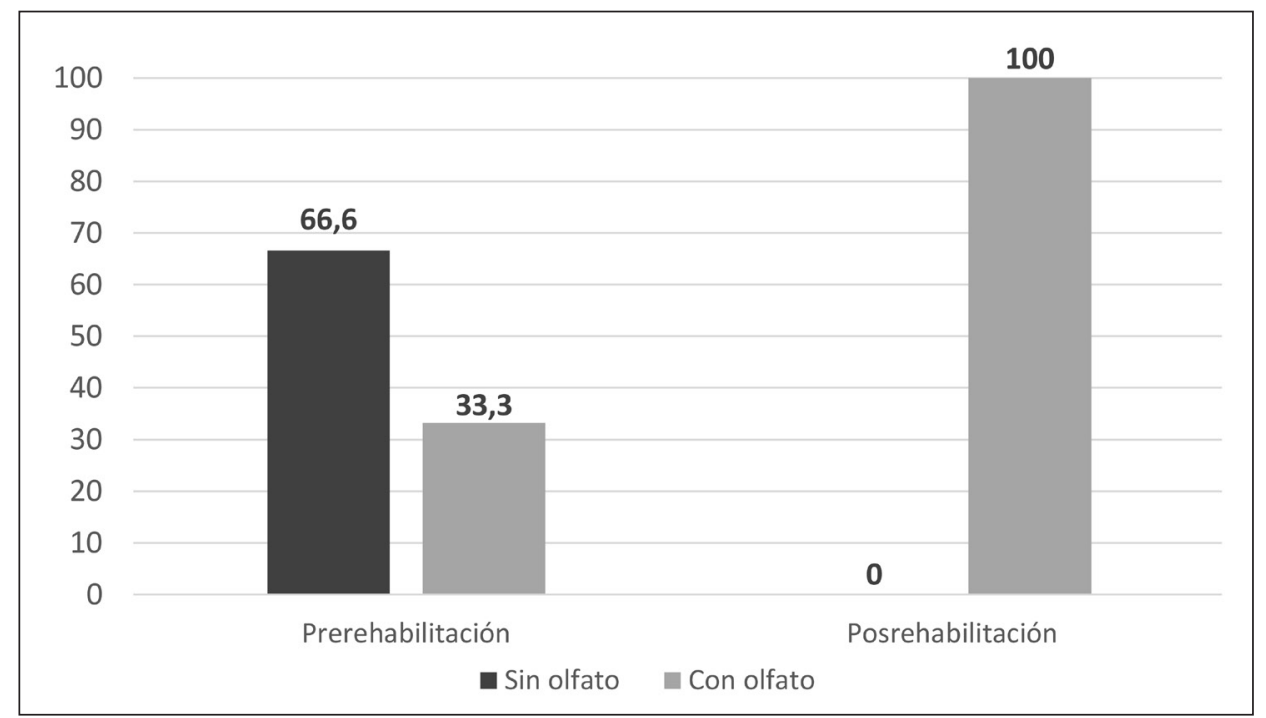

Figura 2. Evaluación del olfato pre y pos rehabilitación con test olfatométrico. olfativa antes de la rehabilitación, tras ésta, les resultaba mucho más fácil percibir el tipo de olor y con mayor intensidad.

\section{Discusión}

Siendo la pérdida de la voz la condición más evidente e incapacitante de una LT, solo un $47 \%$ tenía rehabilitación de la voz. La pérdida del olfato lleva asociada alteraciones del sentido del gusto en mayor o menor medida y expone al paciente a situaciones de riesgo, al ser incapaz de detectar olor a humo, un escape de gas o comida en mal estado, además de crear inseguridad y mayor frustración al no poder percibir el olor corporal frente a situaciones sociales $^{1-3}$.

Al igual que en el resto del mundo, la anosmia en los pacientes con LT es desvalorada y no se considera dentro de la rehabilitación habitual. La población intervenida fue similar a la de otras series en cuanto a sexo y edad. Luego de la LT todos los pacientes quedaron con alteración del olfato, un 66,6\% quedó anósmico, similar a lo descrito en distintas series $(78 \%$, Morales-Puebla y cols. ${ }^{4}$ ). El 33,3\% restante presentó hiposmia probablemente gracias a la adquisición, ya sea de forma autónoma o por entrenamiento, de maniobras de fonación como la erigmofonación.

Posterior a la rehabilitación, el 100\% de los pacientes lograron aplicar la técnica de forma correcta, cinco de ellos en forma automatizada, dentro de estos últimos los cuatro que ya tenían erigmofonación. Ningún paciente tuvo problemas con su adquisición. La rehabilitación del olfato se logró en el 100\% de los pacientes, independiente del tiempo transcurrido desde la cirugía (rango entre 2002-2016). Por lo tanto, que la rehabilitación sea posible sin importar el tiempo que el paciente lleve laringectomizado, implica que el neuroepitelio no se atrofia tras la cirugía ${ }^{8}$.

En la encuesta de percepción del sentido del olfato y del gusto, se objetivó que al mejorar el olfato con la MIFAN, paralelamente mejoró el sentido del gusto, siendo ambos estadísticamente significativo. Todos los pacientes que habían aprendido la técnica MIFAN tenían sensación de mejoría en su calidad de vida, ya que podían apreciar olores en las comidas, en sus casas, en los parques, su olor corporal, perfumes, situaciones cotidianas que antes habían desaparecido para ellos. Es una estimación prometedora de que la MIFAN mejora tanto la percepción del olfato como del gusto en los pacientes laringectomizados y por ende su calidad de vida.

\section{Conclusión}

La MIFAN ha demostrado recuperar el sentido del olfato al restituir el flujo aéreo nasal en 
los pacientes laringectomizados, debiendo ser incorporada en todo protocolo de rehabilitación. Es una técnica de fácil aprendizaje, barata de ejecutar y sin riegos para los pacientes. Si aún no es posible realizar la MIFAN, iniciar precozmente la rehabilitación fonatoria (por ejemplo, la erigmofonación) también mejora el sentido del olfato y del gusto además de preparar el camino para un aprendizaje más rápido y eficaz de la MIFAN.

\section{Bibliografía}

1. Woodard TD, Oplatek A, Petruzzelli GJ. Life after total laryngectomy: a measure of long-term survival, function, and quality of life. Arch Otolaryngol Head Neck Surg. 2007;133:526-532.

2. Moreno AB, Lopes CS. Quality of life assessment in laryngectomized patients: a systematic review. Cad Saude Publica. 2002;18:81-92.

3. Nazar G, Platzer L, González I, Messina A. Evaluación De La Calidad De Vida En Pacientes Tratados Por Cáncer De Laringe. Rev Otorrinolaringol Cir Cabeza Cuello. 2004;64:90-198.
4. Morales-Puebla JM, Morales-Puebla AF, JiménezAntolín JA, Muñoz-Platón E, Padilla-Parrado M, Chacón-Martínez J. Rehabilitación olfativa tras la laringectomía total [Olfactory rehabilitation after total laryngectomy]. Acta Otorrinolaringol Esp. 2010;61(2):128-134. doi: 10.1016/j.otorri.2009.10.011.

5. Hilgers FJ, van Dam FS, Keyzers S, Koster MN, van As CJ, Muller MJ. Rehabilitation of olfaction after laryngectomy by means of a nasal airflow-inducing maneuver: the "polite yawning" technique. Arch Otolaryngol Head Neck Surg. 2000;126(6):726-732. doi: 10.1001/archotol.126.6.726

6. Chacón Martínez J, Morales, Puebla JM, Jiménez Antolín JA. Patología de la olfacción. Olfatometría. Manejo de los problemas olfativos. Libro virtual de formación en ORL 2008; Capítulo 61.

7. Hudson L, Silva MC, Núñez JC, Gómez R, VenegasFrancke P. Valores normales de olfato, hiposmia y anosmia en población chilena sana según la batería "Sniffin Sticks" [Evaluation of olfaction in healthy subjects using the Sniffing Sticks battery]. Rev Med Chile 2012;140(4):442-446. doi: 10.4067/S003498872012000400004 .

8. Fujii M, Fukazawa K, Hatta C, Yasuno H, Sakagami M. Olfactory acuity after total laryngectomy. Chem Senses. 2002;27:117-121. 